\title{
Diclofenac Synthesis
}

\author{
AYMEN LABIDI ${ }^{a, b}$ \\ Organic Chemistry - Organometallic Chemistry \\ Published on Researchgate on January, $25^{\text {th }} 2021$ \\ DOI 10.17605/OSF.IO/F9XWS
}

Diclofenac is an NSAID, Non-Steroidal Anti-Inflammatory Drugs, widely used clinically in the treatement of osteoarthritis, rheumatoid arthritis, and ankylosing spondylitis, and has been available in the United States since 1988 (Banks et al. 1995). Diclofenac can cause hepatotoxicity, and despite the rare nature of these events, affects a large number of people due to its widespread usage. It is one of the most common drugs associated with idiosyncratic hepatic injury (Daly et al. 2007). From November 1988 to June 1991 the FDA received 180 cases of diclofenac-induced adverse hepatic injury of which 79\% were female, $71 \%$ were elderly patients (above the age of 60 ), and $77 \%$ of the patients had osteoarthritis. In most of the cases the liver injury was characterized by hepatocellular damage or mixed hepatocellular injury, and there were some cases of cholestasis (Banks et al. 1995). The incidence of diclofenac hepatotoxicity has been reported to be 6.3 per 100000 patients (DeAbajo et al. 2004). Bioactivation to reactive intermediates, their disposition, the production of oxidative stress, mitochondrial damage, and immune-mediated mechanisms have been suggested to play a role in diclofenac-mediated liver toxicity. 1

\section{Synthesis}

Diclofenac (1.5), 2-[(2,6-dichlorophenyl)-amino]phenylacetic acid, is synthesized from 2-chlorobenzoic acid and 2,6-dichloroaniline. The reaction of these in the presence of sodium hydroxide and copper gives N-(2,6dichlorophenyl)anthranylic acid (1.1), the carboxylic group of which undergoes reduction by lithium aluminum hydride. The resulting 2-[(2,6-dicholorphenyl)-amino]-benzyl alcohol (1.2) undergoes further chlorination by thionyl chloride into 2-[(2,6-dichlorophenyl)-amino]-benzylchloride (1.3) and further, upon reaction with sodium cyanide converts into 2-[(2,6-dicholorophenyl)-amino]benzyl cyanide (1.4). Hydrolysis of the nitrile group leads to diclofenac (1.5) ${ }^{2}$

\section{Types of Chemical Reactions}

The reaction between 2-chlorobenzoic acid and 2,6dichloroaniline is an aromatic nucleophilic substitution called ipso substitution (addition-elimination) so that the product (1.1) is obtained. Lithium aluminium hydrate $\mathrm{LiAlH}_{4}$ commonly abbreviated to LAH, is an inorganic compound is used as a reducing agent in organic synthesis, especially for the reduction of esters, carboxylic acids, and amides, so carboxylic acid of (1.1) will be converted into primary alcohol and a new product will appear (1.2), the resulting primary alcohol (1.2)

${ }^{a}$ University of Carthage, National Institute of Applied Sciences and Technology, Tel. +21697537501; E-Mail: aymenlabidi@insat.u-carthage.tn

${ }^{b}$ Chemical Engineering Student, Chemical Engineering Department

${ }^{c}$ Aeronautical Consultant for FTAA and Glider former and Trainer for Aerobotix INSAT is treated with Thionyl chloride $\mathrm{SOCl}_{2}$ which is an inorganic compound, it is a moderately volatile colourless liquid with an unpleasant acrid odour. Thionyl chloride is primarily used as a chlorinating reagent so that hydroxide group of (1.2) will be converted into Chlorine leading to a new product (1.3). The 2[(2,6-dichlorophenyl)-amino]-benzylchloride (1.3) reacts with sodium Cynanide (Cyanide anion) $\mathrm{NaCN}$ throw a Nuclephilic substitution SN2 reaction mechanism (We have a primary substrate, a very good leaving group and a strong nucleophile) leading to the 2-[(2,6-dicholorophenyl)-amino]benzyl cyanide (1.4), Alkaline Hydrolysis of which using sodium hydroxide leads to a carboxylate salt which reacts with water (Hydron) so that a carboxylic acid will be formed leading the final product: Diclofenac (1.5).

\section{Detailed Mechanism}

The mechanism of diclofenac is detailed in Fig.2, $\mathbf{3}^{\text {rd }}$ page, for the first reaction, which is an ipso aromatic nucleophilic susbtitution, it's a $\mathrm{C}-\mathrm{N}$ coupling, the catalyst used is copper in presence of potassium hydroxyde, as this reaction proceeds well with an electron-rich aryl, it's the Ullmann-type coupling reactions ${ }^{3}$, having observed the improvement using ultrasound in the cross-coupling of o-halobenzoic acids and substituted benzoamines with copper, or $\mathrm{Cu} / \mathrm{Zn}$ under probe sonication ${ }^{3}$, Galy and co-workers successfully synthesized acridine derivatives using this method. In order to replace dry organic solvents with more environmentally benign ones, Pellón et al. extended the synthesis of halobenzoic acid and alkylamine in the presence of water as a solvent with copper under

This journal is @ The Royal Society of Chemistry [year] Journal Name, 2010, [vol], $1-3 \mid 1$ 


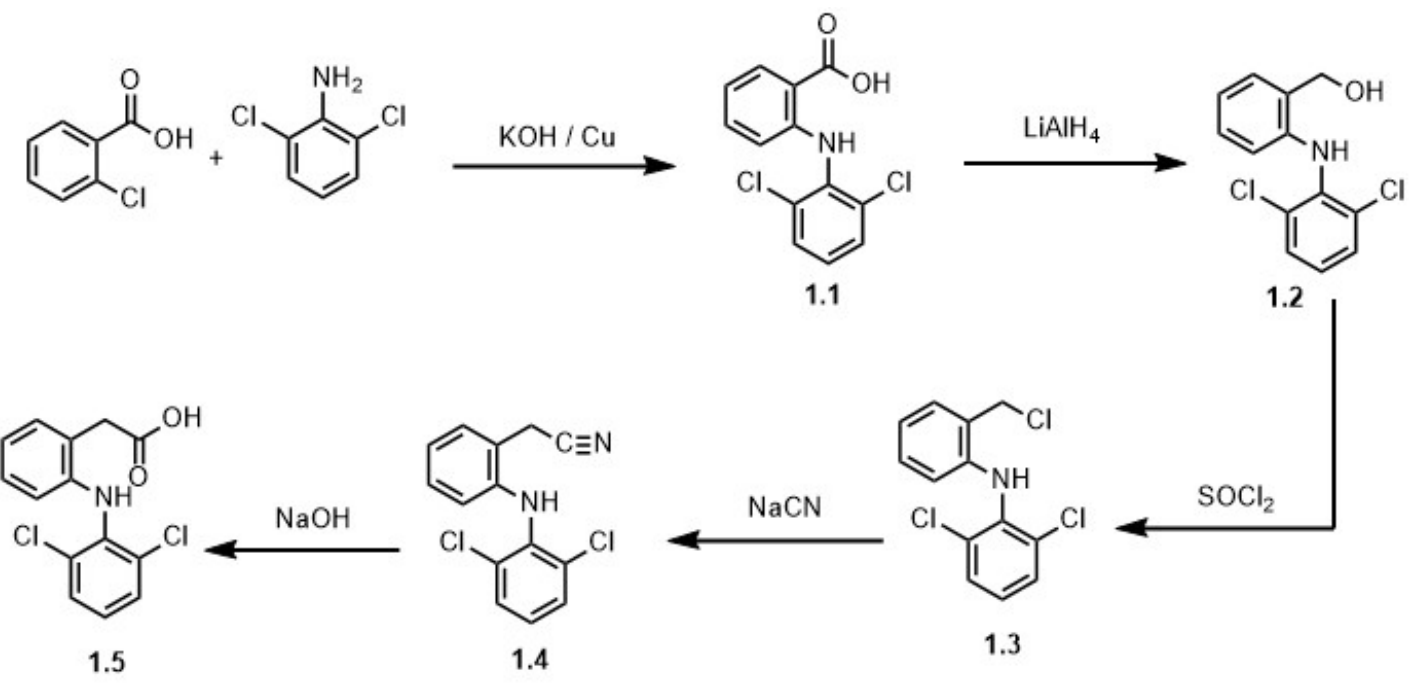

Fig. 1 Synthesis of Diclofenac

probe sonication $^{3}$. In most cases, the yields were $20 \%$ greater than those resulting from classic heating.

From (1.4) to (1.5), there is an enol-ketone Tautomerization equilibrium leading to a carboxylate salt.

\section{Conclusion}

Diclofenac is a widely prescribed nonsteroidal antiinflammatory drug and, after flucloxacillin and amoxicillin-clavulanate, is among the most common drugs causing idiosyncratic DILI. It has been estimated that diclofenacrelated DILI (Drug Induced Liver Injury) occurs in 4 in every 100,000 people prescribed this drug ${ }^{4}$.Susceptibility to diclofenac-related DILI does not appear to relate to HLA genotype ${ }^{4}$.Instead, it has been suggested that reactive metabolites may bind to hepatocyte proteins triggering local damage and inflammation.

\section{References}

1 I. G. Sipes, A. Charlene and A. J. Gandolfi, Comprehensive toxicology, Pergamon Press Oxford, UK, 1997.

2 R. Vardanyan and V. Hruby, Synthesis of essential drugs, Elsevier, 2006.

3 M. Mingos and R. Crabtree, Comprehensive organometallic chemistry III, Elsevier Science, 2007.

4 S. Chackalamannil, D. Rotella and S. Ward, Comprehensive medicinal chemistry III, Elsevier, 2017.

2 Journal Name, 2010, [vol],1-3 


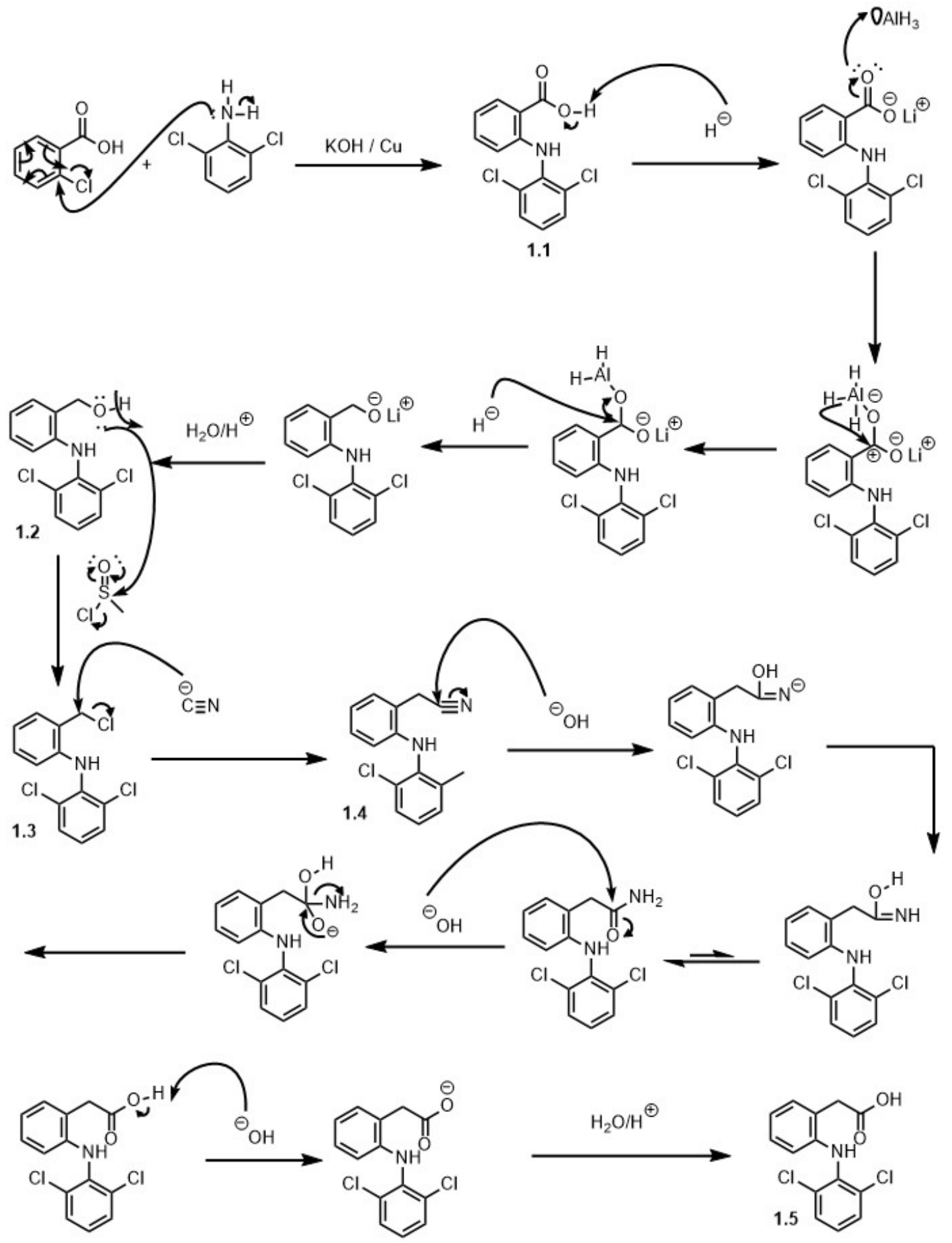

Fig. 2 Mechanism of Diclofenac Synthesis 